Acta Crystallographica Section B

Structural

Science

ISSN 0108-7681

Editor: Sander van Smaalen

Structures of three Schiff-base diazastilbenes: (I) trans- $\mathrm{N}$-(2pyridylmethylene)aniline, (II) trans- $N$-(4-pyridylmethylene)aniline and (III) trans- $\mathrm{N}$-benzylidene-3-pyridinamine

M. Wiebcke and D. Mootz

This electronic document was scanned from an archival copy of material deposited to accompany a paper published in an IUCr journal. In many cases the only accessible copy was a microfilm of a poor-quality original. 
DE POSIT MATERIAL

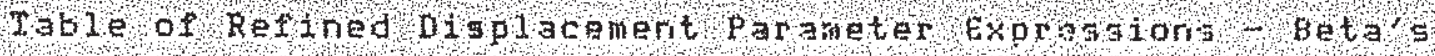

$9 \mathrm{me}$

(1)

(2)

(1)

$1(2)$

$0(3)$

$0(A)$

$0(5)$

$0(6)$

C

$N$
$B(1,1)$

$B(2,2)$

$0.00234(2) 0.00515(5) 0.00240(2)$
$8(1,2)$

$8(1,3)$

$B(2,3)$

$0.00073(3): 0$

$0.00264(4) \quad 0.0080(1) \quad 0.00393(7)-0.0010(1) \quad 0.00208(9) 0.0023(2)$

$0.00559(7) \quad 0.0079(I) \quad 0.00271(5)-0.0039(2) \quad 0.00056(9) 0.0014(1)$

$0.00335(5) \quad 0.0033(1) \quad 0.00165(5)-0.0021(1) \quad 0.0031 .2(8)-0.0054(1)$

$0.00365(5) 0.0088(1) \quad 0.00307(5) \quad 0.0035(2) \quad 0.000 \% 0(9)-0.001 k_{i}(2)$

$0.00320(4) \quad 0.0075(1) \quad 0.00586(6) \quad 0.0003(1) \quad 0.00250(9) 0.0033(2)$

$0.00259(5) \quad 0.0154(2) \quad 0.00284(5) \quad 0.0020(2) \quad 0.00014(8)-0.9042(2)$

$0.00365(3) \quad 0.0075(2) \quad 0.00284(6)-0.0020(2) \quad 0.0022(1) \quad-0.0008(2)$

$0.00365(6) \quad 0.0078(2) \quad 0.00281(5) \quad 0.0013(2) \quad 0.00204(4) 0.000 \%(2)$

The form of the anisotropic displacement parameter is:

exp $(-(B(1, \lambda)+h 2+B(2,2)+k 2+B(3,3)+12+8(1,2) k h k+B(1,3) k h 1$

$+B(2,3)+k i)]$.
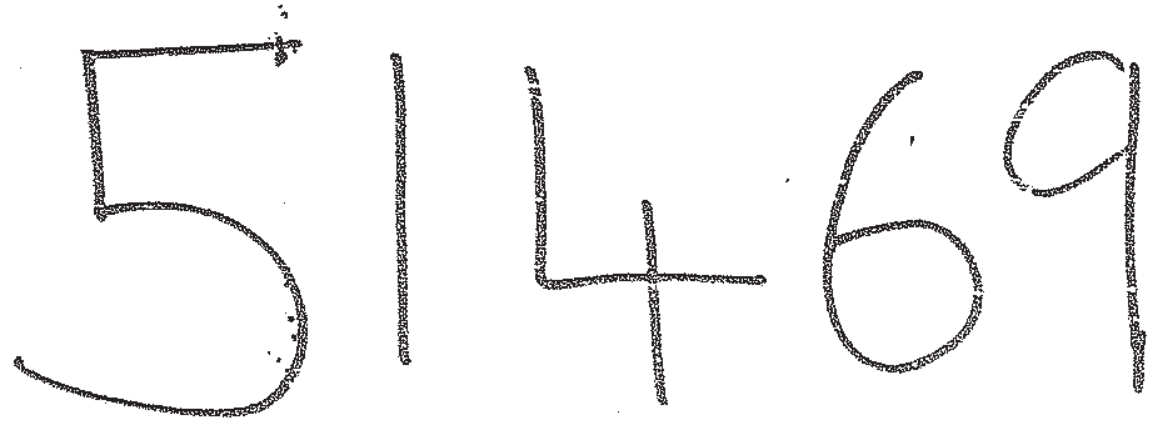


\section{DEPOSIT MATEHTAL.}

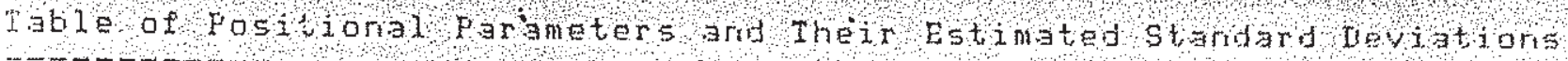

Aton

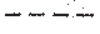

$H(1)$

$H(3)$

$H(4)$

$H(5)$

$H(1 \mathrm{C})$

$H(20)$

H(1)

$H(2+)$

$H(3)$ $\because$.

y

$z$

E (A2)

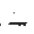

$$
0.345(2)
$$

$$
0.759(3)
$$$$
0.531(6)
$$

$0.223(2)$

$0.014(4)$

$0.011(2)$

$0.639(2)$

$0.098(1)$

$0.054(1)$

$0.400(2)$

$3.317(1)$

$0.626(1)$

$0.874(3)$
$0.365(2)$

$0.246(2)$

$0.531(3)$

$0.380(3)$

$0.026(1)$

$A(1)$

$4.9(6) \%$

$5.0(6) t$

$6 \times 4(7) k$

$3.144)$

$2.4(4) x$

$3.149)$

$2,6(4)+$

$3.1(1)+$

Stared aloms were refined isotrobically. (n) 


\section{Deposit material}

The $\left(\mathrm{NH}_{3}\right)_{-}\left(\mathrm{CH}_{2}\right)_{2}-\left(\mathrm{NH}_{3}\right)$ group

\begin{tabular}{|c|c|c|c|}
\hline $\mathrm{N}-\mathrm{H}(1 \mathrm{~N})$ & $0.75(2)$ & $N(1 N)-N-H(2 N)$ & $105(2)$ \\
\hline$N-H(2 N)$ & $0.84(2)$ & $H(1 N)-N-H(3 N)$ & $110(2)$ \\
\hline $\mathrm{N}-\mathrm{H}(3 \mathrm{~N})$ & $0.87(2)$ & $H(2 N)-N-H(3 N)$ & $106(2)$ \\
\hline & $\because$ & $H(1 C)-C-H(2 C)$ & $103(2)$ \\
\hline $\mathrm{C}-\mathrm{H}(1 \mathrm{C})$ & $0.97(2)$ & $\mathrm{H}(1 \mathrm{C})-\mathrm{C}-\mathrm{C}$ & \\
\hline \multirow[t]{5}{*}{$\mathrm{C}-\mathrm{H}(2 \mathrm{C})$} & $0.92(2)$ & $\mathrm{H}(1 \mathrm{C})-\mathrm{C}-\mathrm{C}$ & $770(1)$ \\
\hline & & $\mathrm{H}(2 \mathrm{C})-\mathrm{C}-\mathrm{C}$ & $115(1)$ \\
\hline & & $H(1 C)-C-N$ & $110(1)$ \\
\hline & & $\mathrm{H}(2 \mathrm{C})-\mathrm{C}-\mathrm{N}$ & $107(1)$ \\
\hline & & $\mathrm{C}-\mathrm{C}-\mathrm{N}$ & $110.5(1)$ \\
\hline
\end{tabular}

$a$ 
Page 1

\begin{tabular}{|c|c|c|c|c|c|c|c|c|c|c|c|}
\hline$H$ & $L$ & tots & Ecto & $H$ & tr & Eobs & tas a & $H$ & $K$ & 2063 & $E c \pm 10$ \\
\hline- & - & $--\infty-\infty$ & $-\cdots-\cdots$ & - & - & $-\cdots-$ & $-\cdots--$ & - & - & $-\cdots-$ & $-\cdots-m-$ \\
\hline 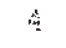 & $0-22$ & $3 y$ & 91 & 10 & $0-1: 3$ & 82 & 30 & 10 & $5-17$ & 33 & $8 B$ \\
\hline 12 & $0-22$ & $\left\{\begin{array}{l}3 \\
0\end{array}\right.$ & 34 & $1\}$ & $0-1 \xi$ & 89 & 85 & 1 & $\because-1 \%$ & $7 \%$ & 76 \\
\hline 4 & $2-22$ & 50 & 60 & 5 & $1-13$ & 58 & 51 & 2 & $0-1 r_{3}$ & $5 \%$ & 51 \\
\hline 3 & $2-2 z$ & 64 & 64 & 2 & $z-1 z$ & 116 & $1) 6$ & $A$ & $0-16$ & 269 & 270 \\
\hline 1 & $3-22$ & 72 & 70 & 10 & $2-13$ & 101 & 94 & 83 & $0-16$ & 120 & 124 \\
\hline 5 & $3-22$ & 67 & 60 & 3 & $3-16$ & 304 & 104 & 10 & $0-16$ & is & 54 \\
\hline$i$ & $1-21$ & 1.14 & 113 & $7^{*}$ & $3-13$ & 35 & $B 1$ & 12 & $0-16$ & 170 & 179 \\
\hline 9 & $1-21$ & 5 & 55 & 13 & $3-14$ & 56 & 52 & 3 & $\therefore-16$ & 58 & 61 \\
\hline 11 & $1-21$ & 61 & 62 & $1 \%$ & $3-23$ & 57 & $5 \%$ & 9 & $1-16$ & 15 & 74 \\
\hline 5 & $3-21$ & 50 & 60 & 滈 & $4-38$ & 63 & 63 & 2 & $2-16$ & s & GI \\
\hline 6 & $A-\therefore 1$ & 63 & 62 & 8 & $1-7.3$ & $5 \%$ & 53 & 1 & $2-1 i$ & 221 & 220 \\
\hline 6 & $5-3$ & 57 & $A 8$ & 10 & $A-\cdots$ & 85 & 84 & 10 & $2-16$ & 28 & 55 \\
\hline 4 & $0-20$ & $\% 3$ & 75 & 12 & $A-1 B$ & 77 & $6 ! 3$ & 12 & 2 . In & $1 \lambda 2$ & 138 \\
\hline 8 & $0-20$ & 95 & 45 & 5 & $5-18$ & 88 & 83 & 16 & $3-16$ & 68 & 66 \\
\hline 10 & $0-20$ & 58 & 53 & $\%$ & $5-18$ & 81 & 82 & 1 & $3-16$ & 72 & 72 \\
\hline 14 & $0-20$ & 31 & 70 & 13 & $5-1\}$ & 58 & a & 3 & $3-26$ & 63 & 64 \\
\hline 16 & $0-30$ & 61 & 6.5 & 1 & $6-18$ & $4 \cdot 3$ & 91 & $y$ & $3-16$ & $1: 30$ & 134 \\
\hline 21 & $1-20$ & 60 & 61 & 12 & $6-18$ & 78 & 71 & 9 & $1-16$ & $10 \%$ & 304 \\
\hline 2 & $2-20$ & 69 & 66 & 11 & $6-13$ & 39 & 35 & 1.5 & $.33+1 r_{3}$ & $7 \%$ & 71 \\
\hline E & $2-20$ & 76 & 30 & 3 & $7-16$ & 71 & 70 & J" & $=3 x-16$ & $\% 1$ & 70 \\
\hline 19 & $2-20$ & if & 51 & 4 & $7-18$ & 89 & $9 \%$ & 4 & $4 \cdots 16$ & 83 & 82 \\
\hline 11 & $3-20$ & $9 A$ & 92 & $i$ & $1-1 \%$ & $6^{A}$ & 65 & 12 & $4-16$ & $6 s$ & 62 \\
\hline 2 & $A-20$ & 63 & 65 & 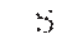 & $1-1.7$ & 59 & 39 & 15 & ì-16 & 6,0 & 60 \\
\hline 4 & $4 \cdots 0$ & 34 & 52 & 7 & $1-1 \%$ & $16 A$ & 162 & I & $3-36$ & $12 \%$ & 123 \\
\hline 10 & $1-20$ & 54 & 54 & 15 & $1-1 \%$ & 145 & 116 & $y$ & 5.5 & 1.56 & 153 \\
\hline 3 & $5-20$ & 65 & 69 & 10 & $\because-1 \%$ & $5 \%$ & 5 & 9 & $5-1 t$ & EEr & 66 \\
\hline 1 & $(1-3)$ & 56 & 53 & 12 & $2-1.7$ & 94 & 93 & $1 \%$ & $5 \cdot 1.0$ & $7 \%$ & 71 \\
\hline 3 & $1-19$ & $8 \%$ & 47 & If & $2-17$ & 60 & 60 & 8 & $6-16$ & $6 \%$ & 63 \\
\hline 5 & $1-12$ & 130 & 131 & 7 & $3-17$ & 1.31 & $1: 13$ & 10 & $5-16$ & 10 & 60 \\
\hline 11 & $1-19$ & 95 & 57 & 25 & $3-17$ & 85 & 86 & 3 & $y-16$ & $1 \% 6$ & 129 \\
\hline 13 & $2-19$ & 90 & 8 A & 2 & $1-1 \%$ & $: 30$ & 79 & 5 & $7-16$ & $6: 3$ & 63 \\
\hline 2 & $2-19$ & 60 & 61 & $A$ & $4-17$ & 142 & 139 & 7 & $7-16$ & $7 n$ & 77 \\
\hline 8 & $2-10$ & 130 & 128 & 8 & $1-17$ & 36 & $3 i$ & 1.1 & $7-16$ & 6.1 & 64 \\
\hline 5 & 3.15 & 76 & 74 & 10 & $4-1 \%$ & 117 & $\pm i v$ & $1 A$ & $8:-16$ & 71 & 75 \\
\hline 1.3 & $3-19$ & $y 1$ & 86 & 13 & $1-1 \%$ & $13 \%$ & 37 & 11 & $9-16$ & 71 & 75 \\
\hline 8 & $4 \cdots 19$ & $11 \mathrm{~A}$ & 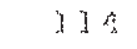 & 1 & $5-17$ & 82 & $\theta^{2}$ & 3 & $1-15$ & 274 & 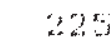 \\
\hline 7 & $5-19$ & 56 & 43 & $: 3$ & $y-1 \%$ & 76 & 71 & 3 & $1 \cdot 12$ & 71 & 73 \\
\hline 8 & $6-19$ & $5 \%$ & 62 & 5 & $5-17$ & $7 \%$ & 72 & 5 & $3 \cdots 15$ & 206 & 200 \\
\hline 12 & $6-19$ & 56 & 92 & $y$ & $5-1 \%$ & 51 & $\because 2$ & 9 & $1-16$ & 10,8 & 162 \\
\hline 2 & $(6-1)$ & 125 & 140 & 1 & $2-17$ & 6 & 65 & $3 \%$ & $3-15$ & 93 & 94 \\
\hline 4 & $0-1\}$ & 78 & $\{3,3$ & 3 & $6-17$ & 76 & 76 & 3 & $\because 1 \because$ & $7 \%$ & 73 \\
\hline 8 & $c-18$ & sis & $\Leftrightarrow / \hat{1}$ & 6 & $6 \cdots 17$ & 106 & $10 \%$ & $A$ & $a \cdots b$ & $\%$ & 76 \\
\hline
\end{tabular}


Page 2

\begin{tabular}{|c|c|c|c|c|c|c|c|c|c|c|c|}
\hline H & $\mathrm{K}^{2} \mathrm{k}$ & Eobs & Ecalc & $\mathrm{H}$ & $k$ & Eobs & $\mathrm{Ecalc}$ & $\mathrm{H}$ & 1 & $\mathrm{Cob}$ & $\mathrm{Ecalc}$ \\
\hline 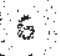 & $2-13$ & 120 & 118 & 16 & $2-11$ & 63 & 61 & 11 & $3-13$ & 56 & 54 \\
\hline$A$ & $2-1 i$ & 89 & 889 & 1 & $3-14$ & 98 & 102 & 13 & $3-13$ & $5 \%$ & 56 \\
\hline 6 & $2-15$ & 19 & $\Omega_{1}$ & 3 & $3-11$ & 135 & 110 & 15 & $3-13$ & 8) & 84 \\
\hline 1 & $3-15$ & $1) 8$ & 118 & 7 & $3-14$ & 85 & 86 & $1 \%$ & $3-13$ & 119 & 119 \\
\hline 9 & $3-15$ & 76 & 71 & 9 & $3-11$ & 61 & 59 & 4 & $1-13$ & 15 & $\lcm{47}$ \\
\hline 13 & $3-15$ & 91 & 90 & 11 & $3-14$ & 128 & 129 & 6 & $4-13$ & 160 & 159 \\
\hline 17 & $3-15$ & 78 & 76 & 15 & $3-14$ & 60 & 5 & 8 & $1-13$ & 134 & 134 \\
\hline 4 & $4-25$ & 123 & 124 & 17 & $3-14$ & 69 & $6 \%$ & $1 \%$ & $4-13$ & 134 & 133 \\
\hline $\begin{array}{r}6 \\
12\end{array}$ & $4-15$ & 138 & 131 & 19 & $3-14$ & 81 & 78 & 16 & $7-13$ & 125 & 125 \\
\hline 12 & $4-15$ & 62 & 63 & 4 & $A-1 A$ & 60 & 64 & 11 & $5-13$ & 54 & 54 \\
\hline 14 & $4-1.5$ & 113 & 112 & 5 & $1-14$ & 59 & 60 & 17 & $5-13$ & $1 / 1$ & 71 \\
\hline 3 & $5-15$ & 110 & 110 & 14 & $4-14$ & 53 & 50 & $A$ & $6-13$ & 15,7 & 156 \\
\hline 5 & $5-15$ & 70 & 70 & 3 & $5-14$ & 183 & 181 & 6 & $6-13$ & 96 & 97 \\
\hline 7 & $5-15$ & 148 & 144 & 9 & $5-14$ & 123 & 122 & 8 & $6-13$ & 149 & 150 \\
\hline 15 & $5-15$ & 66 & 63 & $\mathrm{M}$ & $5-11$ & 123 & 121 & 12 & $5-13$ & 57 & 72 \\
\hline 2 & $6-x 5$ & 98 & 100 & $1 \%$ & $5-14$ & $\theta I$ & sB & 16 & $6-13$ & 86 & 84 \\
\hline 4 & $6-15$ & 275 & 73 & 19 & $5-14$ & 72 & 69 & 3 & $7 \sim 13$ & 98 & 99 \\
\hline 6 & $6-15$ & 120 & 118 & 2 & $6-14$ & 96 & $9 \%$ & 9 & $7-13$ & 82 & 81 \\
\hline 10 & $6-15$ & 72 & 70 & 1 & $6-14$ & 83 & 80 & 13 & $7-13$ & 56 & 60 \\
\hline 14 & $6-15$ & 69 & 73 & 3 & $7-14$ & 122 & 121 & 2 & $8-13$ & 78 & 77 \\
\hline 3 & $7-15$ & 82 & 81 & 7 & $7-14$ & 100 & 101 & 1 & $3-1: 3$ & $12 \%$ & 121 \\
\hline 5 & $7-15$ & 54 & 55 & 9: & $7-14$ & 56 & $5 \%$ & 8 & $8-13$ & 75 & $\begin{array}{r}78 \\
\text { r }\end{array}$ \\
\hline$?$ & $7-15$ & 57 & 59 & 11. & $7-11$ & 83 & 80 & 10 & $3-13$ & 56 & 58 \\
\hline 11 & $7-15$ & 106 & 101 & $8^{\circ}$ & $8-14$ & 59 & 52 & 12 & $8-13$ & 99 & 98 \\
\hline 15 & $7-15$ & 59 & 55 & 5 & $9-11$ & 73 & 72 & 3 & $9-13$ & 71 & 72 \\
\hline 22 & $8-15$ & 85 & 87 & 7 & $9-14$ & 57 & $4 \%$ & $x 3$ & $9-x 3$ & 69 & 69 \\
\hline 8 & $8-15$ & 105 & 101 & is & $9-14$ & 6.5 & 61 & 2 & $10-13$ & 61 & 63 \\
\hline 10 & $8-15$ & 80 & 74 & 3 & $x-13$ & 334 & 338 & 10 & $10-13$ & 70 & 69 \\
\hline 11 & $9-15$ & 69 & 65 & 5 & $1-13$ & 166 & 169 & 12 & $10-13$ & 81 & 78 \\
\hline 2 & $0-14$ & $19 \%$ & $19 A$ & $\%$ & $1-13$ & 60 & 66 & $\%$ & $11-13$ & 61 & 2 \\
\hline 6 & $0-11$ & 306 & 303 & 9 & $1-13$ & 72 & 75 & 2 & $0-12$ & 221 & 438 \\
\hline 8 & $0-I 4$ & 113 & 216 & I & $1-13^{6}$ & 205 & $20 \%$ & 6 & $0-12$ & 136 & 133 \\
\hline 10 & $0-11$ & 67 & 68 & 13 & $1-13$ & 136 & 139 & 8 & $0-12$ & 161 & 164 \\
\hline 14 & $0-14$ & 225 & 226 & 35 & $3-13$ & 102 & 98 & 10 & $.0-12$ & $2 \% 4$ & 285 \\
\hline 16 & $0-11$ & $7: 3$ & 75 & 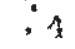 & $2-13$ & 65 & 64 & 14 & $0-12$ & 59 & 61 \\
\hline 1 & $2-14$ & 50 & 51 & 6 & $2-13$ & 73 & 69 & 16 & $0-12$ & II 4 & 117 \\
\hline 3 & $x-14$ & 76 & $1 / 6$. & 8 & $2-1: 3$ & 119 & 115 & $1.8^{\circ}$ & $0-12$ & B8 & 90 \\
\hline 7 & $1-14$ & 61 & 63 & 10 & $2-13$ & $1 A 8$ & 147 & 4 & $1-12$ & $A A$ & 47 \\
\hline 11 & $1-1 \mid$ & 62 & 63 & 11 & $2-1: 3$ & 36 & 39 & 5 & $1 \cdot 1 \%$ & 125 & 118 \\
\hline 13 & $1-14$ & 80 & 82 & 16 & $2-13$ & 55 & 60 & 7 & $1-12$ & 155 & 134 \\
\hline 6 & $2-11$ & 203 & 207 & 3 & $3-13$ & 151 & $16 c_{3}$ & 9 & 1. -12 & $8: 3$ & 86 \\
\hline 14 & $z-14$ & 171 & $1 / 6$ & 7 & $3-13$ & 162 & 169 & 31 & $1-12$ & 60 & 63 \\
\hline
\end{tabular}


$2804)(\mathrm{HSP} 208)(\mathrm{NH} 3)(\mathrm{CH} 2) 2(\mathrm{NH} 3)$

\begin{tabular}{|c|c|c|c|c|c|c|c|c|c|c|c|}
\hline 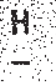 & $\mathrm{K}$ & Fobs & $\mathrm{ECalC}$ & $\mathrm{H}^{-}$ & $\mathrm{K}^{\prime} \mathrm{l}$ & $806 s$ & $\mathrm{Egalc}$ & $\mathrm{H}$ & k 1 & tobt & $\mathrm{Ecal}^{\circ}$ \\
\hline . & $2-12$ & 129 & 127 & 9 & $9-12$ & 138 & $13 \%$ & 2 & $3-11$ & 115 & 115 \\
\hline 8 & $2-12$ & 234. & $2 A 1$ & 11 & $1-12$ & 53 & 46 & 6 & $8-11$ & 115 & 113 \\
\hline 6 & $2-12$ & 105 & 103 & 60 & $2-12$ & 64 & 64 & 10 & $8-11$ & 67 & G6 \\
\hline 8 & $2-12$ & 78 & 76 & 1 & $1-11$ & 49 & 46 & 14 & $8-11$ & 12 & 74 \\
\hline 0 & $2-12$ & 185 & 185 & 5 & $1-11$ & 230 & 233 & 3 & $9-11$ & 55 & 48 \\
\hline & $2-12$ & 77 & 87 & $z$ & $1-11$ & 329 & 328 & 4 & $10-11$ & 72 & 72 \\
\hline & $2-12$ & 67 & 63 & 8 & $1-11$ & 60 & 61 & 6 & $10-11$ & 119 & 115 \\
\hline 16 & $2-12$ & 76 & 16 & 13 & $1-11$ & 199 & 196 & 3 & $11-11$ & 81 & 84 \\
\hline 8 & $2-32$ & 92 & 92 & 15 & $1-31$ & $1 \% 6$ & $17 \%$ & 11 & $11-11$ & 66 & 65 \\
\hline 1 & $3-32$ & 86 & 88 & 19 & $1-11$ & 58 & 60 & $\therefore$ & $0-10$ & 321 & 318 \\
\hline 3 & $3-12$ & 126 & 128 & $2 i$ & $1-11$ & 59 & 57 & 1 & $0-10$ & 211 & 246 \\
\hline 5 & $3-12$ & $166^{\circ}$ & 168 & 2 & $2-11$ & 319 & 325 & 6. & $0-10$ & 111 & 112 \\
\hline 7 & $3-12$ & 81 & 79 & 1 & $2-11$ & 63 & 63 & 8 & $0-10$ & 140 & 139 \\
\hline & $3-12$ & 132 & 128 & 6 & $2-11$ & 169 & 167 & 10 & $0-10$ & 58 & 52 \\
\hline 1 & $3-12$ & 62 & 62 & 8. & $2-11$ & 153 & 158 & 12 & $0-10$ & 321 & 316 \\
\hline 21 & $3-12$ & 69 & 67 & 10 & $2-11$ & $\because 74$ & 74 & 14 & $0-10$ & 126 & 122 \\
\hline 4 & $4-12$ & 170 & 179 & $12 \%$ & $2-11$ & 16 & 41 & 16 & $0-10$ & 121 & 122 \\
\hline 14 & $4-12$ & 92 & 90 & 14 & $2-11$ & 44 & 39 & 20 & $0-10$ & 132 & 128 \\
\hline 1 & $5-12$ & 161 & 165 & 18 & $2-11$ & 82 & 81 & 1 & $1-10$ & 93 & 89 \\
\hline 3 & $5-22$ & 79 & 80 & $\frac{1}{2}$ & $3-11$ & 190 & 195 & 3 & $1-10$ & 98 & 102 \\
\hline 5 & $5-12$ & 156 & 158 & 5 & $3-11$ & 92 & 90 & 5 & $1-10$ & 185 & 187 \\
\hline 7 & $5-12$ & 50 & 48 & $7^{\circ}$ & $3-11$ & 163 & $I \in I$ & 9 & $1-10$ & 43 & 37 \\
\hline 9 & $5-12$ & 77 & 93 & 7 & $3-11$ & 77 & 82 & 13 & $1-10$ & 46 & 46 \\
\hline 13 & $5-12$ & 164 & $1 \% 2$ & 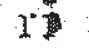 & $3-11$ & 80 & 82 & 15 & $1-10$ & . 35 & 57 \\
\hline 2 & $6-12$ & 19 & 18 & 13 & $3-11$ & 88 & 91 & 2 & $2-10$ & 197 & 191 \\
\hline 4 & $6-12$ & 75 & 71 & 15. & $3-11$ & 106 & $\because 05$ & $\Leftrightarrow$ & $2-30$ & 235 & 2817 \\
\hline 6 & $6-12$ & 98 & 91 & 2 & $1-11$ & $18 \%$ & 193 & 6 & $\because 2-10$ & 161 & 164 \\
\hline 10 & $6-12$ & 50 & 47 & 10 & $4-12$ & 228 & 228 & 8 & $2-10$ & $2 \triangle 4$ & 244 \\
\hline 1 & $7-12$ & 159 & 167 & 12 & $1-11$ & 81 & 80 & 12 & $2-10$ & 239 & 240 \\
\hline 5 & $7-12$ & 164 & 166 & 24 & $\alpha-1 I$ & 54 & 53 & 16 & $2-10$ & 83 & 79 \\
\hline 7 & $7-12$ & 51 & لّا & 13 & $1-11$ & 80 & 80 & 20 & $2-10$ & 9.3 & 89 \\
\hline 9 & $7-12$ & 70 & 71 & 20 & $4-11$ & 71 & $1 / 3$ & 1 & $3-10$ & 62 & 60 \\
\hline 13 & $7-12$ & 92 & 92 & 1 & $3-11$ & 53 & 50 & 3 & $: 3-10$ & 1.11 & 114 \\
\hline 2 & $8-12$ & 110 & 111 & 7 & $5-x$ & $4 y$ & An & 5 & $3-10$ & 143 & 146 \\
\hline 1 & $8-12$ & 70 & 69 & 2 & $6-1 \mid$ & 157 & 133 & 7 & $: 3-10$ & 205 & 205 \\
\hline 6 & $8-12$ & 93 & 9 & 4 & $6-11$ & 142 & 139 & 9 & $3-10$ & 168 & 172 \\
\hline 10 & $8-12$ & 71 & 70 & 10 & $6-11$ & 181 & $1 \% \%$ & 11 & $3-10$ & \%月 & 72 \\
\hline 12 & $8-12$ & $7 \%$ & $7 \%$ & 14 & $6-11$ & 114 & 308 & 15 & $3-10$ & 93 & 32 \\
\hline 1 & $9-12$ & 97 & 100 & 18 & $6-11$ & $6 H$ & 73 & 17 & $3-10$ & 108 & 104 \\
\hline 3 & $9-12$ & 62 & 62 & 1 & $7-11$ & 81 & 77 & $A$ & $4-10$ & $11 \mathrm{~A}$ & 110 \\
\hline 5 & $9-12$ & 50 & $x y$ & 3 & $7-1.1$ & 82 & 32 & $G_{3}$ & $1-10$ & 255 & 262 \\
\hline r & $9-12$ & 89 & 88 & 13 & $7-11$. & 91 & 43 & $\theta$ & $4-10$ & 108 & 122 \\
\hline
\end{tabular}


$P \cap 36,4$

\begin{tabular}{|c|c|c|c|c|c|c|c|c|c|c|c|c|c|}
\hline H & $\mathrm{KL} L$ & Eobs & $\mathrm{Fcalc}$ & 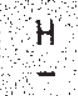 & $k$ & 1 & Eobs & $\mathrm{ECa} 1 \mathrm{c}$ & 4 & 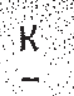 & 1 & $\begin{array}{l}t a b \\
-\end{array}$ & $E C \rightarrow 1 C$ \\
\hline 34 & $4-10$ & 82 & 80 & 15 & 1 & -9 & 59 & 59 & 1 & 9 & -9 & 112 & 142 \\
\hline 16 & $4-10$ & 70 & 70 & 17 & 1 & -9. & 192 & 189 & 3 & 9 & -9 & 58 & 58 \\
\hline 8 & $1-10$ & 66 & 68 & 19 & 1 & -9 & 59 & 35 & 7 & 9 & -9 & 61 & 59 \\
\hline 3 & $5-10$ & 141 & 143 & 2 & 2 & -9 & 140 & $1 A G$ & 2 & 10 & -9 & 93 & 89 \\
\hline 7 & $5-10$ & 211 & 239 & 1 & 2 & -9 & 101 & 108 & 8 & 10 & -9 & 110 & 108 \\
\hline 9 & $5-10$ & 123 & 125 & 6 & 2 & -9 & 211 & 208 & 10 & 10 & -9 & 70 & 67 \\
\hline 1 & $5-10$ & 155 & 153 & 10 & 2 & -9 & 78 & 81 & 5 & 11 & -9 & 104 & 104 \\
\hline 5 & $5-10$ & 84 & 86 & 12 & 2 & -9 & 152 & 153 & 9 & Q1 & -9 & 54 & 52 \\
\hline 17 & $5-10$ & 115 & 111 & 1 & 3 & -9 & 330 & 321 & 2 & 0 & $-B$ & 183 & 481 \\
\hline 2 & $6-10$ & 109 & 105 & 。 & 3 & -9 & 226 & $23 \%$ & 4 & 0 & -8 & 78 & 73 \\
\hline 6 & $6-10$ & 106 & 110 & 9 & 3 & -9 & 251 & 269. & 6 & 0 & -8 & 388 & 390 \\
\hline 10 & $6-10$ & 133 & 129 & 11 . & 3 & -9 & 79 & 82 & 10 & 0 & $-\theta$ & 60 & 57 \\
\hline 12 & $6-10$ & 79 & 770 & 17 & 3 & -9 & 119 & 118 & 12 & $0:$ & -8 & $9 \%$ & 96 \\
\hline 16 & $6-10$ & 68 & 68 & 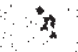 & 4 & -9 & 280 & 280 & 14 & 0 & -8 & 228 & 226 \\
\hline 1 & $7-10$ & 107 & 104 & 6 & 1 & -9 & 319 & 311 & 15 & 0 & -3 & 74 & 69 \\
\hline 3 & $7-10$ & 82 & 83 & 12 & 4 & -9 & 141 & 137 & 22 & 0 & -8 & 125 & 117 \\
\hline 5 & $7-10$ & 67 & 687 & 11 & 1 & -9 & 152 & 150 & 3 & 1 & -13 & $\% 2$ & 72 \\
\hline 7 & $7-10$ & 111 & 110 & 26 & 4 & -9 & 88 & 83 & 5 & 1 & -8 & (6) & 59 \\
\hline 11 & $7-10$ & 147 & 146 & 20 & 4 & -9 & 71 & 69. & 淬 & 1. & -3 & 68 & 67 \\
\hline 13 & $7-10$ & 61 & 59 & 1 & 5 & -9 & 105 & 102 & 9 & 1 & -8 & 184 & 182 \\
\hline 15 & $7-10$ & 76 & 175 & 3 & 5 & -9 & $1 B \%$ & 195 & 11. & 1 & -8 & 22 & 96 \\
\hline 19 & $7-10$ & 69 & 68 & 5 & 5 & -9 & 64 & $\%$ & 13 & 1. & -8 & 49 & 50 \\
\hline 2 & $8-10$ & 138 & 134 & 9 & 5 & -9 & 11 & 11 & 19 & 1 & -8 & 61 & 65 \\
\hline 4 & $8-10$ & 84 & 78 & 1,1 & 5 & -3 & 103 & 105 & 2 & 2 & $-n$ & 102 & 96 \\
\hline 6 & $8-10$ & 70 & 72 & 13 & 5 & -9 & 35 & 51 & 5. & 2 & $-B$ & 1621 & 465 \\
\hline 10 & $0-10$ & 141 & 141 & .2 & G. & -9 & 66 & 66 & 8 & 2 & -4 & $17 \%$ & 179 \\
\hline 1 & $8-10$ & 90 & $3 \%$ & 1 & 6 & -9 & 112 & 138 & 12 & 2 & $f_{13}$ & 15 & $A A_{A}$ \\
\hline 3 & $9-10$ & 188 & 185 & 6 & $G$ & -9 & 136 & 133 & IA & 2 & -8 & 143 & 179 \\
\hline 5 & $9-10$ & 69 & 72 & 10 & 6 & -9 & 189 & 87 & 13 & 2 & -8 & $\% 4$ & 69 \\
\hline 11 & $9-10$ & 212 & 108 & 12 & 6 & -9 & 89 & 89 & 22 & 2 & -8 & 68 & 69 \\
\hline 13 & $9-10$ & 90 & 90 & 14 & 6 & -9 & 116 & 114 & 1 & 3. & -9 & 301 & 304 \\
\hline 2 & $10-10$ & 99 & 94 & 20 & 6 & -9 & 75 & 71 & 3 & 3 & -8 & 295 & 291 \\
\hline 8 & $10-10$ & 55 & 52 & 1 & $y$ & -9 & 129 & 122 & 3 & 3 & -13 & 58 & .69 \\
\hline 12 & $10-10$ & 71 & 12 & 3 & 7 & -9 & 103 & 101 & 9 & 3 & -8 & 314 & 308 \\
\hline 3 & $11-10$ & 72 & 73 & 5 & 7 & -9 & $5 \%$ & 51 & 11 & 3 & $-B$ & 63 & 65 \\
\hline 9 & $I]-10$ & 61 & 64 & 7 & 7 & -9 & 94 & 96 & 17 & 3 & -8 & 65 & 68 \\
\hline 8 & $22-10$ & 71 & 72 & 2 & 8 & -9 & 120 & 122 & 19 & 3 & -8 & 94 & 9 \\
\hline 1 & $1 .-9$ & 345 & $34 \%$ & $A$ & 8 & -4 & 71 & 68 & 2 & 4 & $-B$ & 90 & 9 \\
\hline 3 & $\begin{array}{cc}1 & -y\end{array}$ & 113 & 115 & $\cdot 6$ & 8 & -9 & 83 & 31 & 1 & 1 & -3 & $6: 5$ & 69 \\
\hline 5 & $\begin{array}{lll}1 & -5\end{array}$ & 50 & 13 & \& & 8 & -9 & 94 & 43 & 6 & 4 & -8 & $168^{\circ}$ & 170 \\
\hline 7 & $1-9$ & 123 & 128 & 1.10 & b & -6 & 99 & 102 & 10 & 1 & -8 & $89^{\circ}$ & 9 \\
\hline 9 & $1-9$ & 480 & $18 \%$ & 12 & 8 & $\ldots . .9$ & 60 & 60 & 24 & A & -8 & 99 & \\
\hline
\end{tabular}




\section{(6PO4) (45P208) (NH3) (CH2) 2 (NH3)}

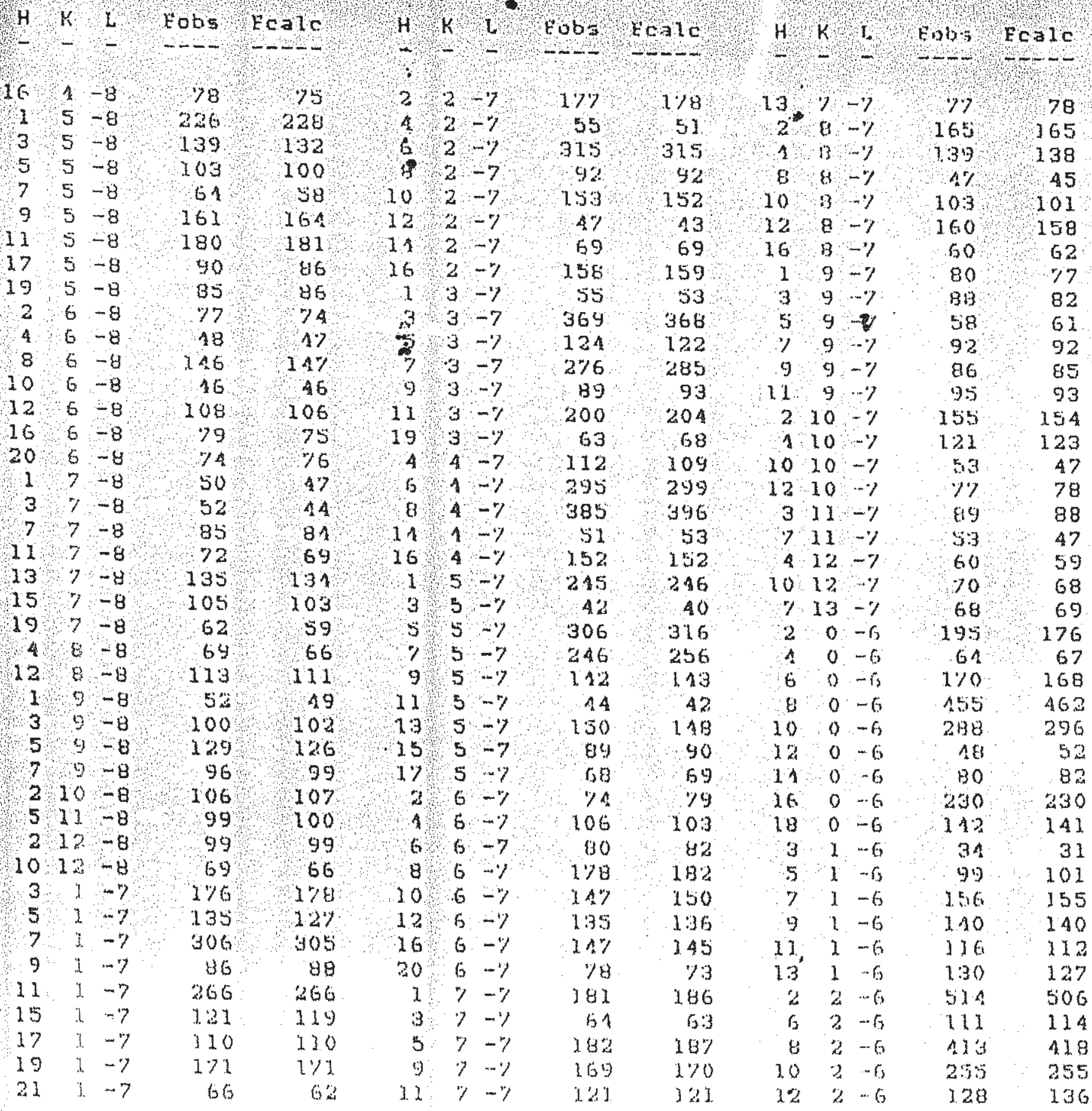


$04)(H 5 P 208)\left(\mathrm{NH}_{3}\right)(\mathrm{CH} 2) 2(\mathrm{HH})$

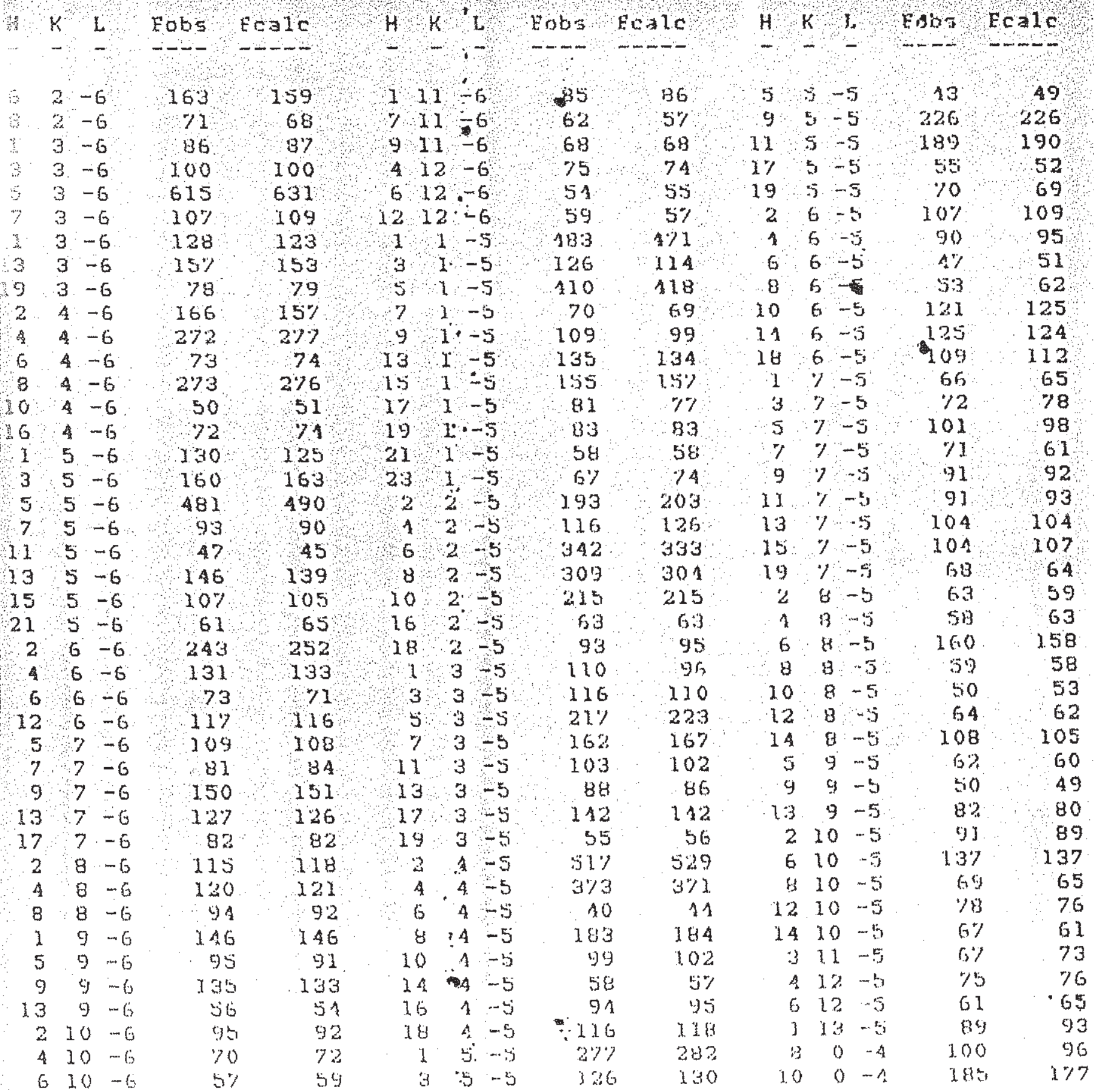




\section{W $2 F 04)(\mathrm{HSP} 208$ (NH3) (CH2) 2 (NH3)}

$\mathrm{P}=9 \mathrm{e} 7$

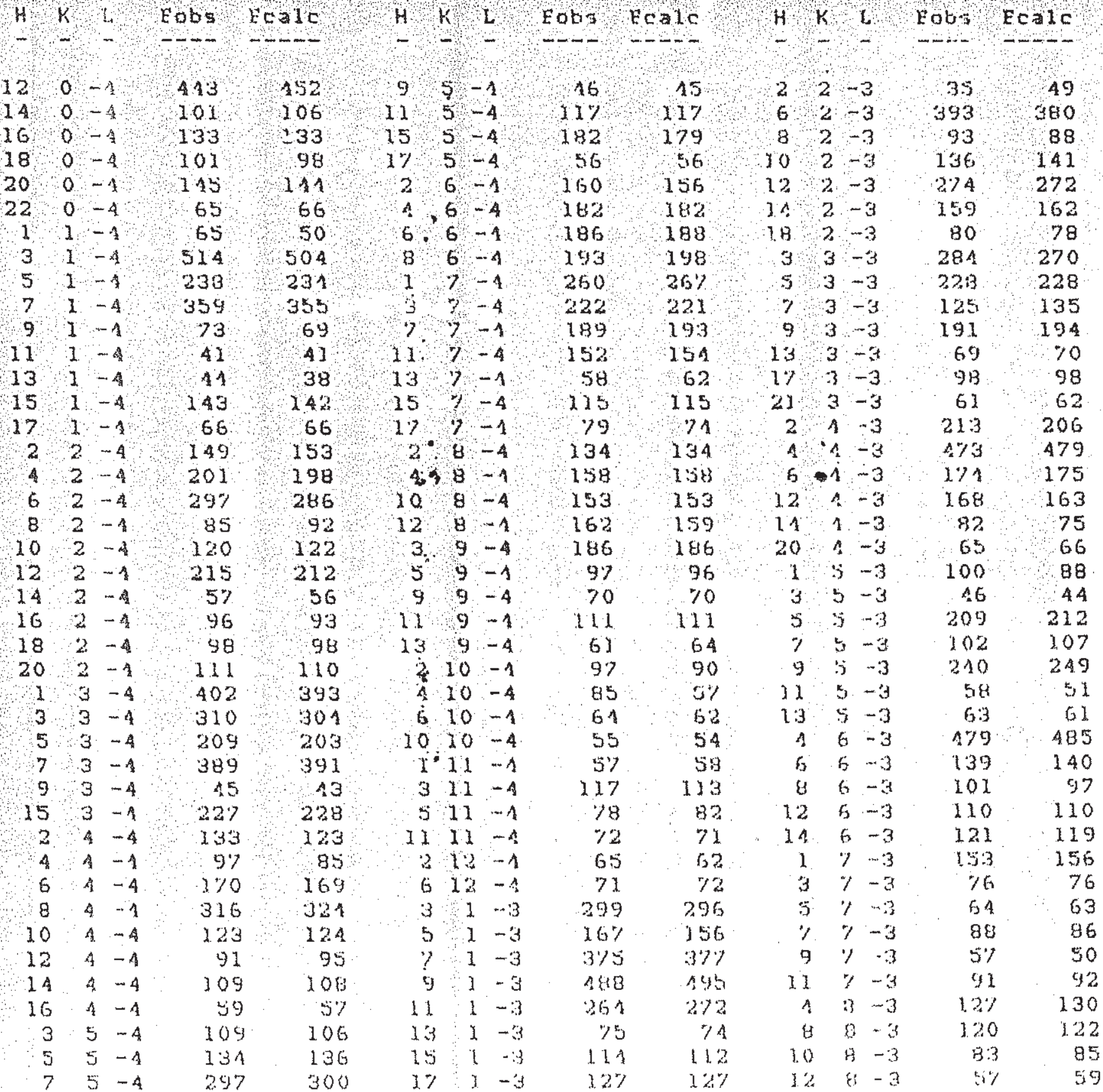


$\mathrm{Page} 8$

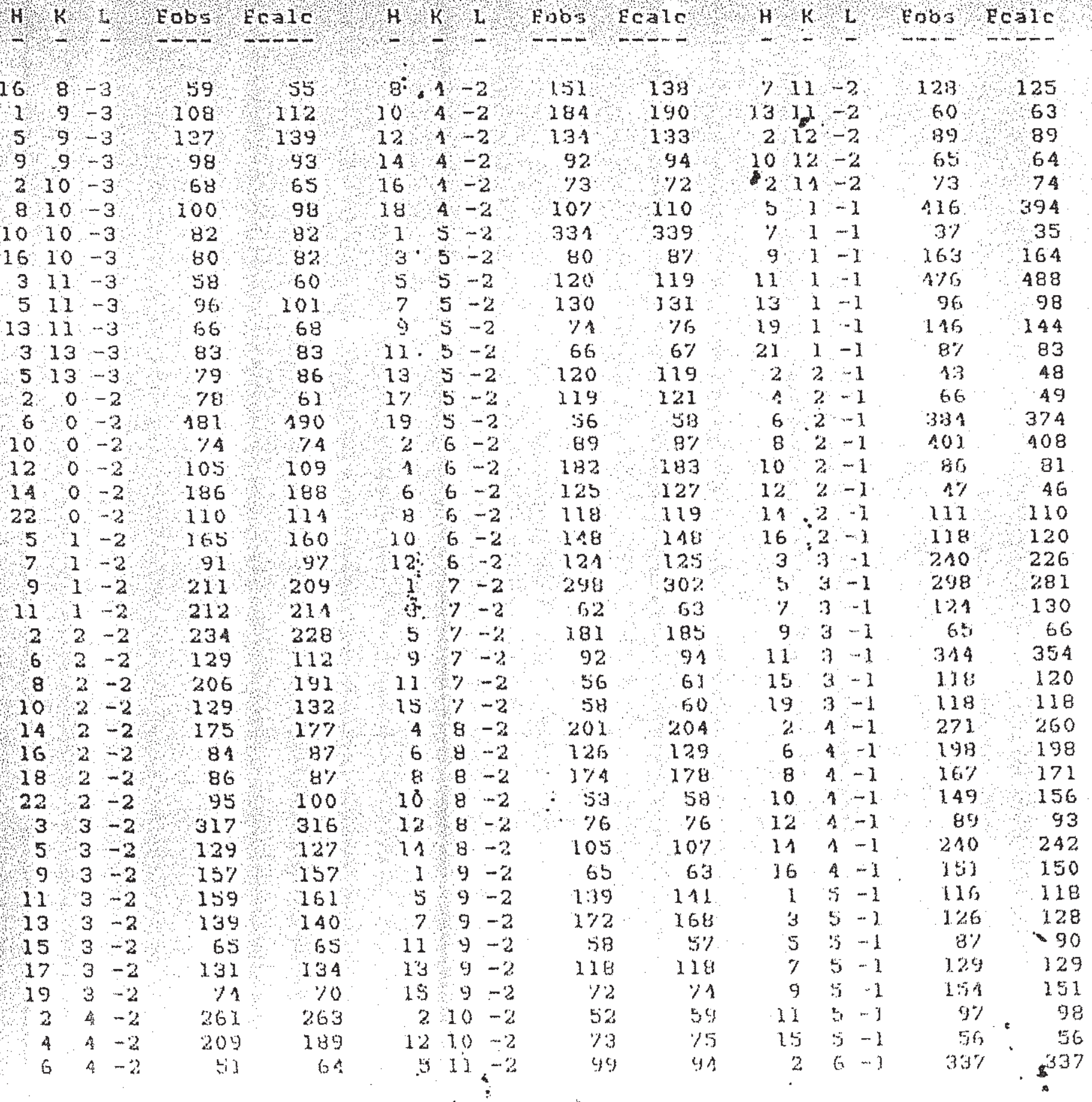


Rage 9

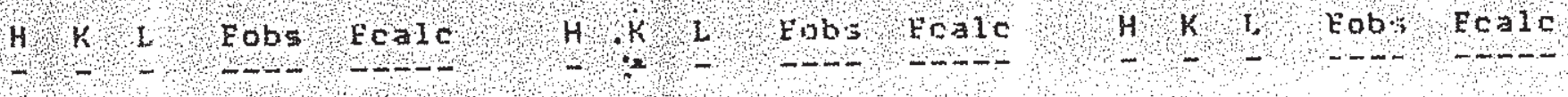

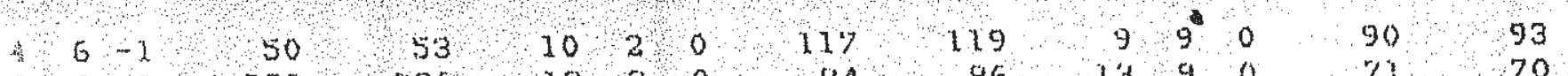

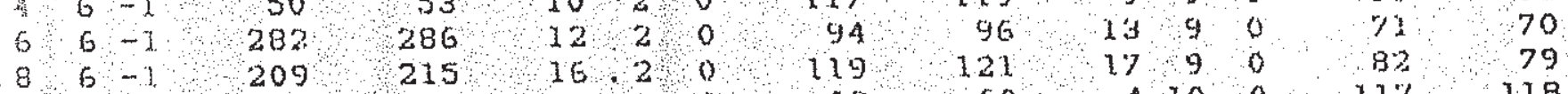

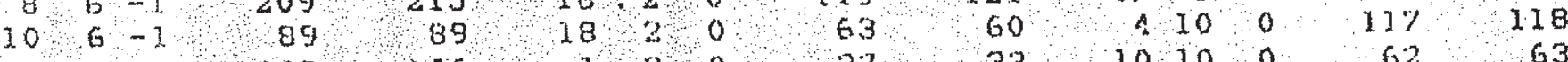

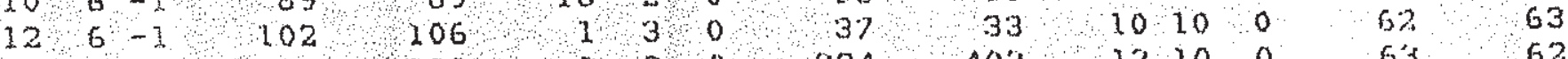

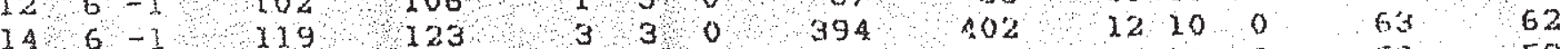

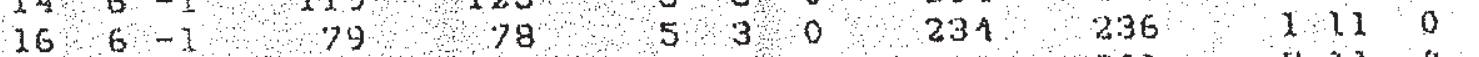

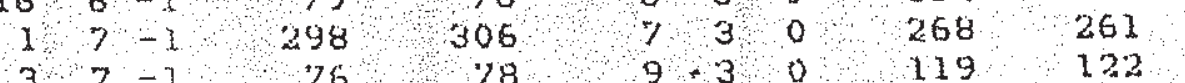

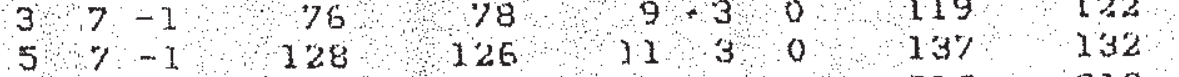

$7110 \quad 52 \quad 55$

$9110 \quad 63 \quad 70$

$120155 \quad 157$

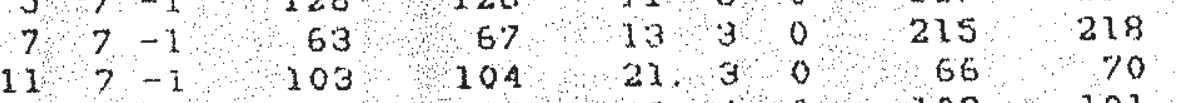

$31 \quad 1 \quad 23 \% \quad 235$

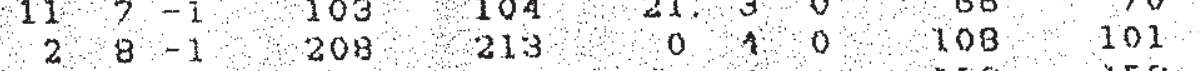

$511 \quad 122 \quad 116$

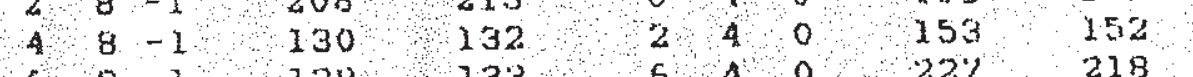

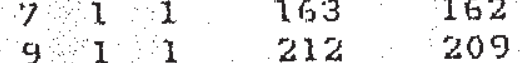

$\begin{array}{ccccccc}6 & 8 & -1 \\ 10 & 8-1\end{array}$

$13112 \quad 246 \quad 246$

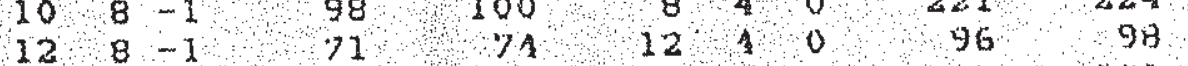

$21 \quad 1$

$109 \quad 109$

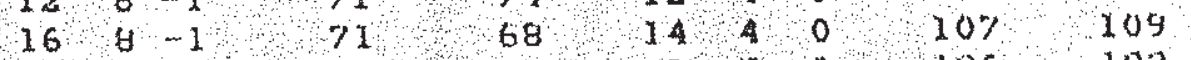

2,21

39 $\quad 46$

$350 \quad 553$

$\begin{array}{lllllll}1 & 9-1 \\ 3 & 9-1 & 1\end{array}$

6 2 I

$145 \quad 140$

$\begin{array}{lll}3 & 9 & -1 \\ 5 & 9 & -1\end{array}$

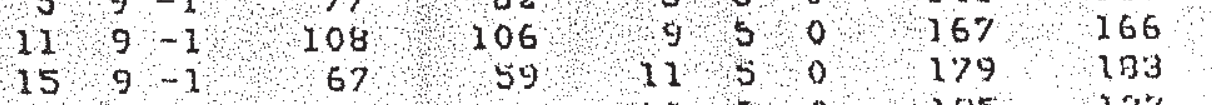

$82^{2} \quad 155 \quad 151$

102 16 $203 \quad 207$

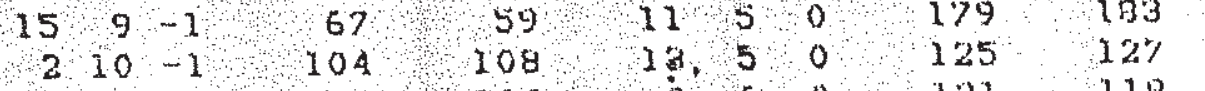

13,21

$\% \quad 78$

$185 \quad 179$

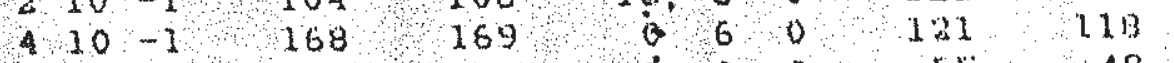

1331

$278 \quad 272$

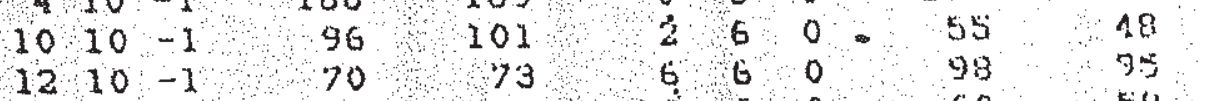

$5,3 \quad 1$

$98 \div 97$

$186 \because 172$

$511-1270075010,60060 \% 58$

$73 \quad 1$

$150 \quad 245$

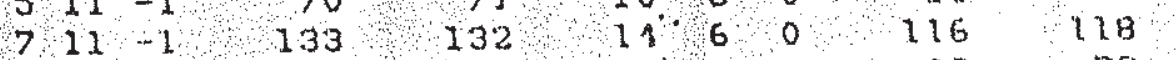

931

$99 \quad 97$

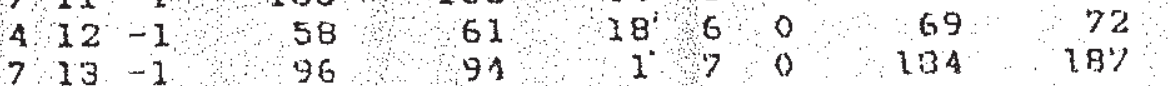

1131

$15 \% \quad 156$

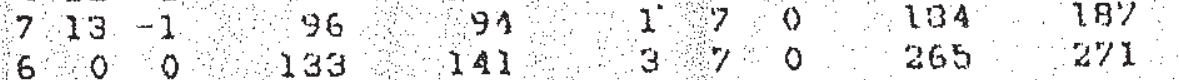

$\begin{array}{ll}3.3 & 1 \\ 15 \cdot 3 & 1\end{array}$

B2 84

80000511 517 $54700311 \quad 320$

19. 3

$60 \quad 65$

$10000330 \quad 330 \quad \cdots 7700055053$

$21 \div 3: 1$

$73 \quad 75$

$16 \quad 0 \quad 0 \quad 88 \quad 887$

$9170 \quad 126$

126

0.41

$55 \quad 55$

$130 \quad 133$

211

$\begin{array}{lr}37 & 29 \\ 4 & 41\end{array}$

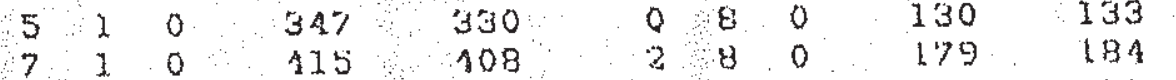

$6 \quad 4 \cdot 1$

41
188

910063

63

72

12

1041

$2 \% 3$

277

10.130 115 146

If 1

75

77

$54 \quad 58$

$18 \& 3$

148

150

$143 \quad 148$

99

100

$220 \quad 100$

79

122

124

$\begin{array}{lll}1 & 5 & 1 \\ 3 & 5 & 1\end{array}$

404

405 
Page 10

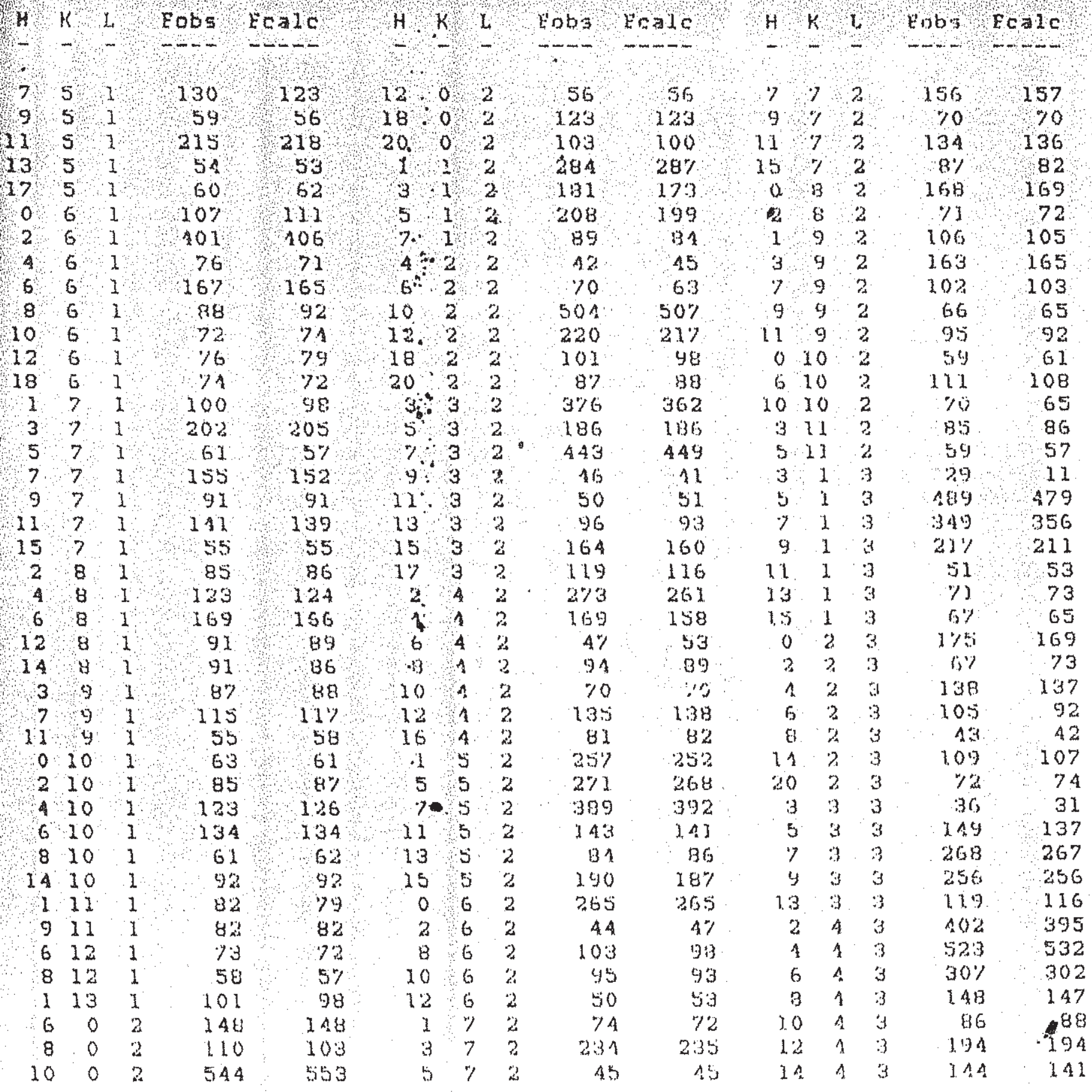




\section{F04 $45 F 208)(N H 3)(C H 2) 2\left(N_{3}\right)$}

$P=9 e 1$

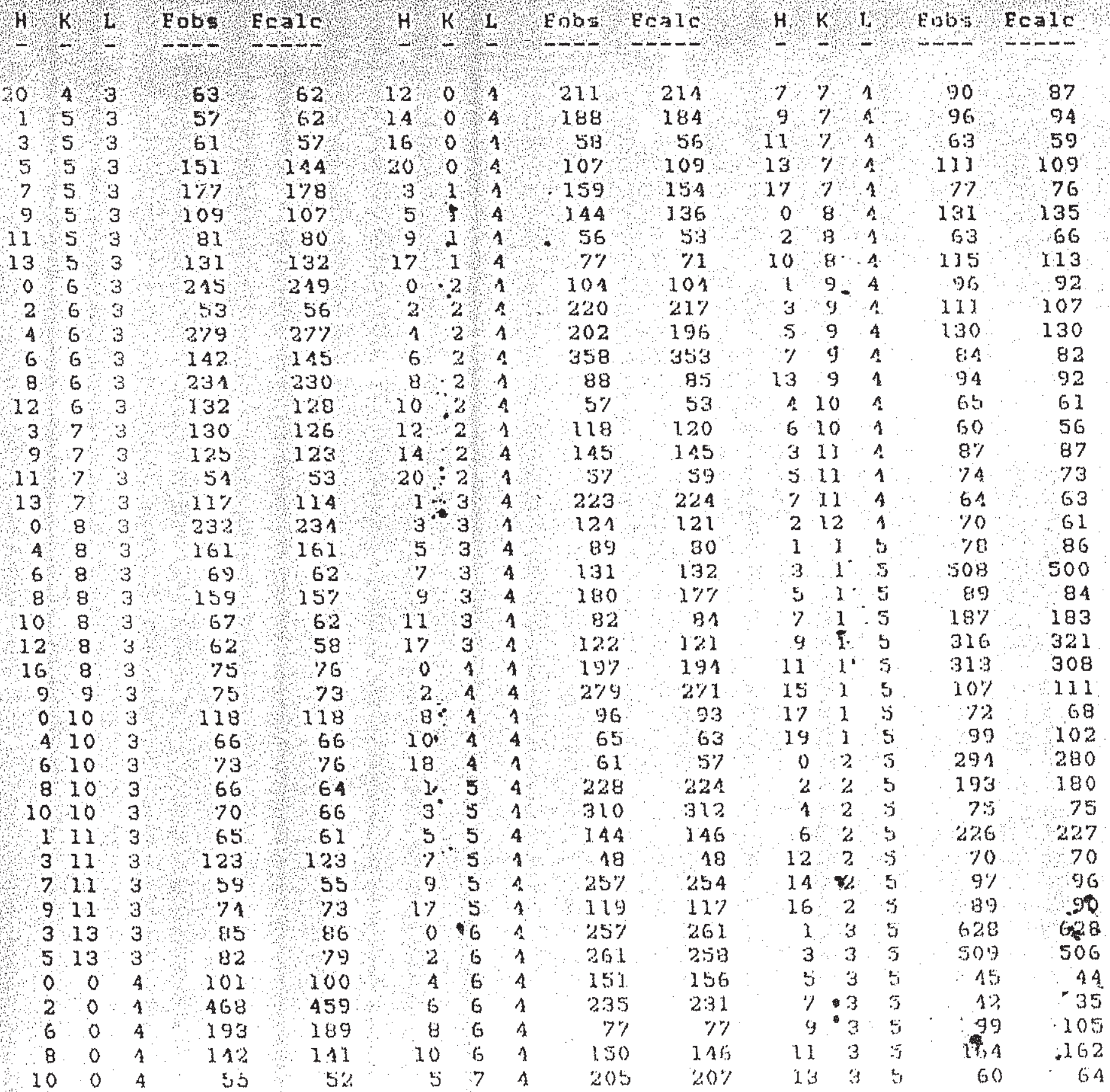




\section{(2POA) (HEP2OB) (NH3) (CH 2$) 2(\mathrm{NH} 3)$}

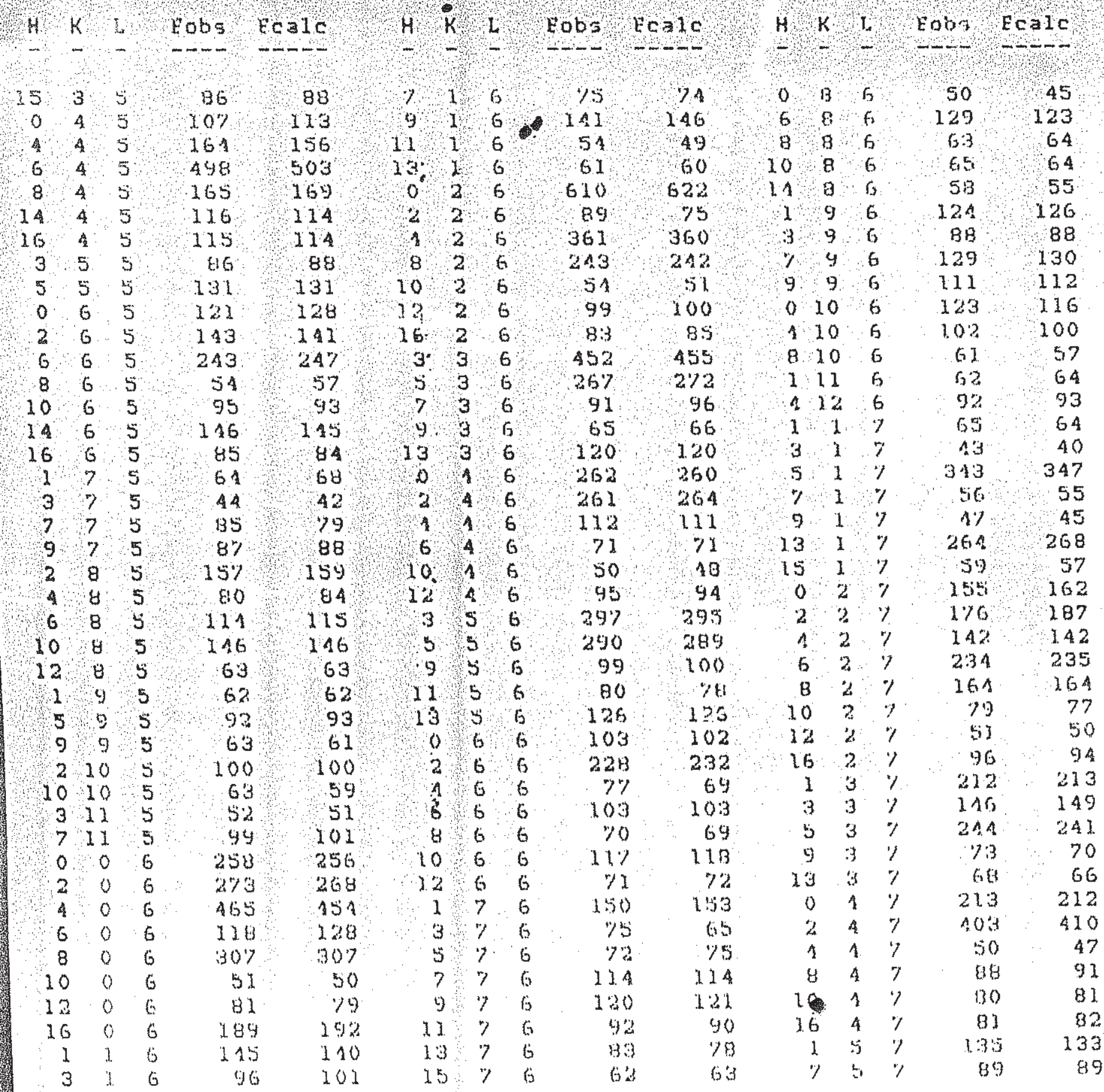


Page 13

\begin{tabular}{|c|c|c|c|c|c|c|c|c|c|c|c|c|c|c|}
\hline 4 & $\mathrm{~K}$ & $L$ & Eobs & $\mathrm{Ecal} 1 \mathrm{C}$ & $\mathrm{H}$ & $k$ & 1 & $8 \supset b s$ & $\mathrm{Eoatc}$ & H & $k$ & 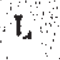 & Eob & $\mathrm{ECA} 1 \mathrm{C}$ \\
\hline 9 & 5 & 7 & 83 & 83 & 14 & $a$ & 8 & 76 & $9 \%$ & $\%$ & 1 & 9 & 213 & 219 \\
\hline 2 & 6 & 7 & 135 & 139 & 1 & 3 & 8 & 47 & 44 & 9 & 1 & 9 & 183 & 187 \\
\hline 6 & 6 & 7 & 75 & 84 & $\mathrm{~s}$ & 3 & 13 & 283 & 287 & 13 & 1 & 9 & 67 & 66 \\
\hline 8 & 6 & 7 & 130 & 130 & 7 & 3 & 8 & 136 & 138 & 0 & 2 & 9 & 54 & 51 \\
\hline 10 & 6 & 7 & 118 & 117 & 11 & 3 & 8 & 19 & 16 & 2 & 2 & 9 & 255 & 256 \\
\hline 16 & 6 & 7 & 12 & 873 & 13 & 0 & 8 & 109 & 102 & 4 & 2 & 9 & 345 & 344 \\
\hline 1 & 7 & 7 & 115 & 112 & 15 & 3 & 8 & 79 & 80 & 10 & 2 & 9 & $10 \%$ & 108 \\
\hline 3 & 7 & 7 & 275 & 48 & 0 & 4 & 8 & 43 & 100 & 12 & 2 & 9 & 110 & 110 \\
\hline 5 & 7 & 7 & 69 & 20 & 2 & 1 & 13 & 302 & 299 & 3 & 3 & 9 & 242 & 245 \\
\hline 9 & 7 & 7 & Eil & 54 & 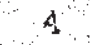 & $A$ & 8 & 53 & 55 & 5 & 3 & 9 & 179 & 187 \\
\hline 11 & 7 & 7 & 95 & 91 & 6 & 1 & 9 & 129 & 130 & 11 & 3 & 9 & 61 & 60 \\
\hline 0 & 8 & 7 & 44 & 38 & 10 & 4 & घ) & $7 A$ & GS & 13 & 3 & 9 & 66 & 61 \\
\hline 4 & 8 & 7 & 110 & 112 & 3 & 5 & 8 & 101 & 103 & 0 & 4 & 9 & 290 & 298 \\
\hline 6 & 8 & $?$ & 122 & $1] 8$ & 5 & 5 & 8. & 2.22 & 210 & 2 & a & 9 & IIs & 109 \\
\hline 10 & 8 & 7 & 66 & 653 & 7 & $\$$ & 0 & 115 & 119 & 1 & 4 & 3 & 195 & 199 \\
\hline 12 & 8 & 7 & 56 & $5 \%$ & 11 & $y$ & 4 & 76 & 73 & $y$ & 4 & 9 & 50 & 52 \\
\hline 14 & 8 & 7 & 65 & $b A$ & 13 & $s$ & 8 & $\$ 1$ & 50 & 12 & 1 & 9 & 122 & 118 \\
\hline 3 & 9 & 7 & 19 & 75 & 15 & 5 & 4 & $1) 4$ & 110 & 1 & 5 & 9 & 130 & 131 \\
\hline 5 & 9 & 7 & $\mathrm{BI}$ & 61 & 8 & 6 & 3 & 159 & $6 \%$ & 3 & 5 & 9 & 216 & 113 \\
\hline 0 & 10 & 7 & 55 & 54 & 16 & $\theta$ & $\varepsilon$ & 55 & 53 & 5 & 5 & 9 & $8 \%$ & 90 \\
\hline 4 & 10 & 7 & 112 & 112 & 1 & $y$ & 8 & 53 & 54 & 7 & 5 & 9 & 38 & 93 \\
\hline 6 & 10 & 7 & 893 & 89 & 5 & 7 & 8 & $13 \%$ & 137 & 9 & 5 & 9 & 51 & 54 \\
\hline 1 & 11 & 7 & 110 & 109 & 7 & 7 & B) & 145 & 111 & 11 & 5 & 9 & 38 & 59 \\
\hline 1 & 13 & 7 & 63 & 61 & 11 & 1 & $y:$ & 91 & 96 & 13 & 5 & $y$ & 73 & 66 \\
\hline 0 & 0 & $b$ & 309 & 315 & 15 & 8 & 8 & 139 & 43 & 0 & 6 & 5 & 101 & 107 \\
\hline 2 & 0 & 8 & 478 & 483 & 0 & 8 & $\theta$ & 62 & 63 & 2 & 6 & y & I\%9 & 168 \\
\hline 4 & 0 & $B$ & 111 & 103 & 3 & 8 & 8 & 116 & 115 & 1 & is & 9 & 139 & 137 \\
\hline 6 & 0 & 8 & 56 & $\$ 6$ & 8 & 1 & $\Leftrightarrow$ & 145 & 143 & $\theta$ & $G$ & $y$ & $1 / 5$ & 76 \\
\hline 10 & 0 & 8 & 359 & 361 & 10 & $\theta$ & 8 & 171 & 75 & 12 & $G$ & 9 & 1.35 & 134 \\
\hline 12 & 0 & 8 & 193 & 191 & 12 & 8 & 8. & $5 \%$ & 58 & 2 & 7 & 4 & 133 & 134 \\
\hline 14 & 0 & $B$ & 109 & 110 & 1 & 9 & 8 & 120 & 120 & 5 & 7 & 9 & 173 & 170 \\
\hline 18 & 0 & 8 & 82 & 80 & 3 & 9 & $B$ & $1 \times 8$ & 117 & g) & $\%$ & 9 & 105 & 102 \\
\hline 3 & 1 & $B$ & 118 & 151 & 5 & 5 & $B$ & 51 & 46 & 0 & B & 9 & 139 & 136 \\
\hline 5 & 1 & 8 & 250 & 255 & 9 & 8 & $\theta$ & 64 & 64 & $A$ & 8 & 9 & 94 & 90 \\
\hline 7 & 1 & $\theta$ & $1 \perp 1$ & 116 & 11 & 9 & 3 & 90 & 85 & $B$ & 日 & 9 & 121 & 123 \\
\hline 13 & 1. & $\xi$ & 91 & $\therefore 89$ & 6 & 10 & 8 & 80 & 45 & 1 & 9 & 9 & 113 & 112 \\
\hline 2 & 2 & 3 & 480 & 490 & 1 & 11 & 8 & 61 & 58 & 3 & 9 & 9 & 71 & 72 \\
\hline 4 & 2 & 8 & 49 & 43 & 3 & 11 & $\theta$ & 56 & 50 & 5 & 4 & 9 & 01 & 64 \\
\hline 6 & 3 & $B$ & 80 & 90 & 6 & 12 & $\theta$ & 61 & 6.5 & 7 & 9 & 9 & $B 3$ & 80 \\
\hline 8 & 2 & 8 & 45 & 43 & 1 & I & 9 & 152 & $14 \%$ & $y$ & 9 & 9 & BO & 19 \\
\hline 10 & 2 & 8 & $1 \% 3$ & 168 & 3 & 1 & 9 & 1.22 & 130 & 0 & 10 & $\mathrm{r}$ & $1.3 \%$ & 138 \\
\hline 12 & 2 & 8 & 74 & $1 / 4$ & 5 & 1 & 9 & 66 & 62 & 2 & 10 & 9 & 乼 & 93 \\
\hline
\end{tabular}


$2 \mathrm{PO} 4)(\mathrm{MSP} 208)(\mathrm{NH} 3)(\mathrm{CH} 2) 2 \mathrm{NH} 3)$

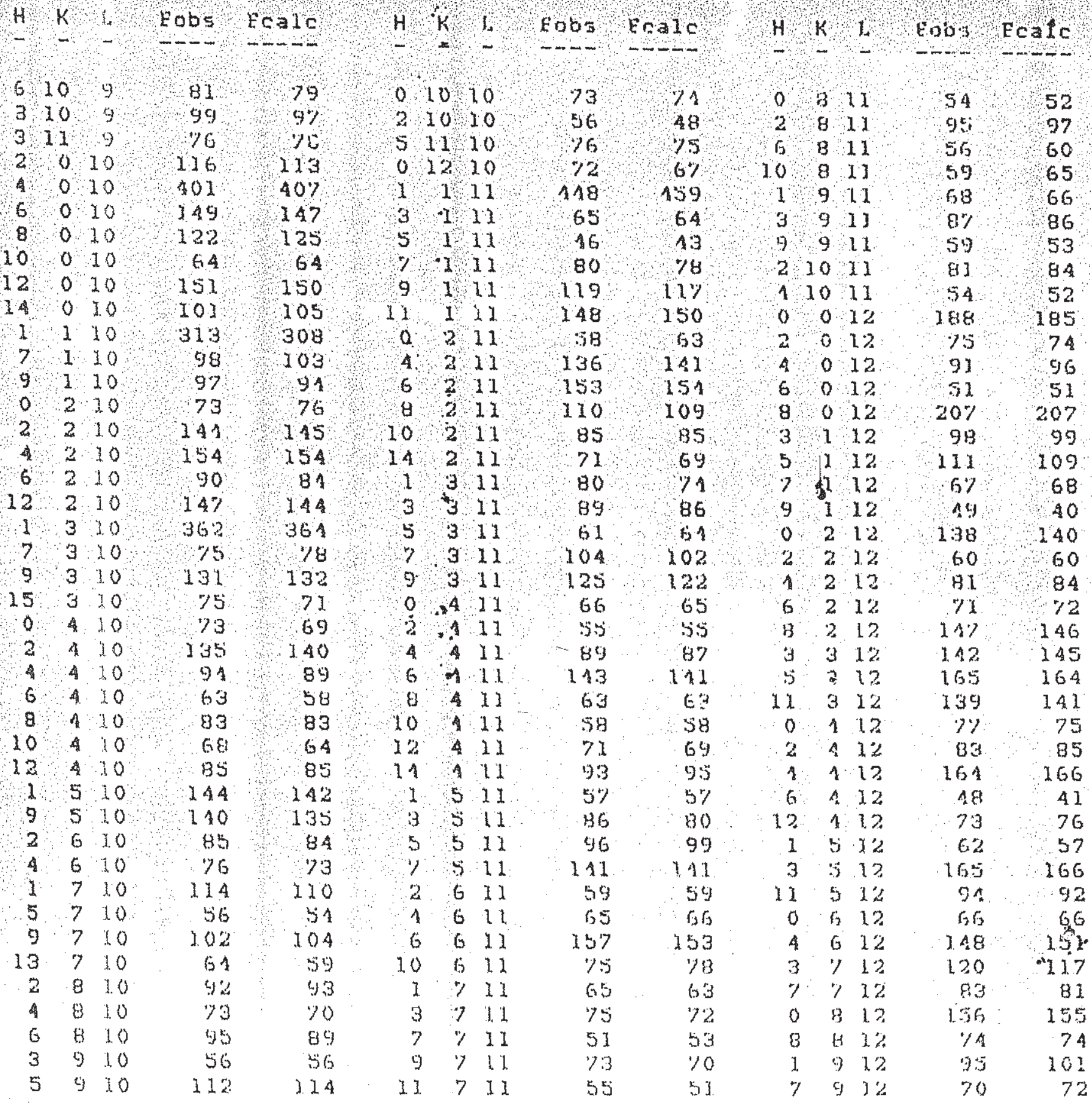


Page 15

\begin{tabular}{|c|c|c|c|c|c|c|c|c|c|c|c|c|}
\hline$k$ & $\mathrm{~L}$ & Eobs & $\mathrm{Ecalc}$ & $\mathrm{H}$ & $\mathrm{K} \mathrm{L}^{\mathrm{L}}$ & Cobs & $E \subset \rightarrow 10$ & $H$ & $k$ & 1 & $8 \Delta b=$ & $\mathrm{ECA} 1 \mathrm{c}$ \\
\hline & -1 & & ----- & - & $=$ & ---- & ---- & - & - & - & ---- & $-r-$ \\
\hline 10 & 12 & $5 \%$ & 57 & 6 & 111 & 106 & 111 & 1 & 11 & $1 \%$ & 151 & 150 \\
\hline 11 & 12 & 58 & $\leqslant 9$ & 10 & 414 & 55 & 58 & 3 & 1 & 17 & 97 & 75 \\
\hline 1 & 33 & 50 & 52 & 5 & 511 & 111 & 138 & 5 & 1 & 17 & $\% 1$ & 70 \\
\hline 3 & 13 & 118. & 118 & 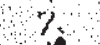 & 514 & 201 & 101 & 9 & 1 & $1 \%$ & 78 & 80 \\
\hline 5 & 13 & 181 & 179 & 0 & 611 & 114 & 113 & 0 & 2 & $1 \%$ & 19 & 52 \\
\hline & 13 & 70 & 71 & 4 & \&IA & 64 & 67 & 4 & 2 & 17 & 54 & 49 \\
\hline 0. & 13 & 122 & 121 & 6 & 611 & 39 & 95 & 1 & 3 & 17 & $\% 8$ & 74 \\
\hline 2 & 13 & 152 & 152 & 8 & 611 & 86 & 87 & $\%$ & 3 & 17 & 65 & 65 \\
\hline 6 & 13 & 60 & 62 & 1 & 711 & 103 & 106 & 1 & 4 & 17 & 69 & 64 \\
\hline 8 & 13 & 96 & 99 & 4 & 711 & 84 & 85 & 6 & 4 & $1 \%$ & 93 & 89 \\
\hline 3 & 13 & 59 & 59 & 9 & 714 & 56 & 51 & 7 & ثئ. & 17 & 61 & 62 \\
\hline 5 & 3. 13 & 123 & 121 & 4 & $\theta \quad 24$ & 62 & 54 & 2 & 6 & $1 \%$ & 60 & 63 \\
\hline 9 & 3:13: & 57 & 58 & 1 & 911 & 65 & 65 & 6 & 6 & $1 \%$ & 11 & 72 \\
\hline 11 & 313 & 62 & 60 & 3 & 115 & 70 & 66 & 3 & $\%$ & $1 \%$ & 58 & 60 \\
\hline 3 & 313 & $7 \%$ & 79 & 7 & 115 & 159 & 150 & 2 & 9 & $1 \%$ & 65 & 67 \\
\hline 0 & 13 & 156 & $1 S B$ & 4 & 215 & 92 & 95 & 0 & 0 & 18 & 125 & 123 \\
\hline 2 & 413 & 26 & 100 & 12 & 15 & 51 & AB & b & 0 & 18 & 125 & 125 \\
\hline 4 & 13 & 13 & 14 & $\%$ & 15 & 119 & $11 \%$ & 8 & 0 & 18 & EI & 66 \\
\hline 8 & 413 & 161 & 162 & 0 & 15 & 1,2 & 60 & 0 & 2 & 18 & 72 & 69 \\
\hline 0 & 413 & 62 & 67 & 2 & 115 & $x 29$ & 125 & 4 & 2 & 18 & 59 & 59 \\
\hline 1 & 513 & 115 & 116 & 1 & 115 & 155 & 1.56 & 6 & $?$ & 18 & 31 & 81 \\
\hline 9 & 13 & 83 & 91 & 10 & 415 & 66 & 66 & 1 & 3 & $1 \varepsilon$ & 59 & 52 \\
\hline 0 & $13:$ & 178 & $1 \%$ & 3 & 515 & 91 & 96 & 3. & 3 & 18 & B? & 86 \\
\hline 8 & 613 & 63 & 60 & 3 & 515 & 84 & 81 & 4 & 4 & 36 & $5 \%$ & 58 \\
\hline 1 & $7 \quad 13$ & 55 & 62 & 0 & 615 & 113 & $11: 3$ & 3 & 5 & 18 & $12 \%$ & 122 \\
\hline 4 & 813 & 79 & 79 & 2 & 615 & 43 & 82 & 3 & $\gamma$ & 18 & 66 & 70 \\
\hline 41 & 013 & 65 & 62 & 1 & 15 & 92 & 97 & 1 & $h$ & 19 & 59 & 57 \\
\hline 0 & 014 & 101 & 165 & 6 & 615 & 61 & 65 & 3 & 2 & 19 & 98 & 95 \\
\hline 2 & 014 & 167 & 170 & 12 & y 15 & 65 & 6.5 & 5 & 1 & 19 & 75 & 74 \\
\hline 6 & 014 & 94 & 97 & 0 & 8 Is & 93 & 95 & 1 & 3. & 19 & 80 & 76 \\
\hline 10 & 014 & 73 & 74 & 2 & 016 & 89 & 88 & 0 & 1 & 19 & 9.5 & 94 \\
\hline 3 & 114 & 45 & 41 & 4 & $0 I G$ & $18 \%$ & 187 & 0 & 6 & 25 & 99 & 96 \\
\hline 0 & 214 & 108 & 107 & 3 & 1 16 & 36 & 54 & 0 & 0 & 20 & 39 & 53 \\
\hline 2 & 214 & 145 & 142 & 4 & 216 & 262 & 158 & 'z & 0 & 20 & 124 & 123 \\
\hline 6 & 214 & 73 & 172 & 1 & 316 & $13 B$ & 90 & 2 & 2 & 21 & 86 & 81 \\
\hline 8 & 214 & 61 & 64 & 3 & 316 & 79 & 82 & & & & & \\
\hline 10 & 214 & 107 & $10 \%$ & 7 & 316 & 14 & 73 & & & & & \\
\hline 5 & is 14 & 109 & 107 & 10 & 416 & 61 & 61 & & & & & . \\
\hline 7 & 311 & 108 & 116 & 1 & 516 & 126 & 122 & & & & & $\cdots$ \\
\hline 13 & 314 & 56 & 51 & 7 & 516 & 58 & 62 & & & & & ; \\
\hline 0 & 411 & 48 & 52 & 2 & $\Leftrightarrow 16$ & 70 & 67 & & & & & \\
\hline 2 & 114 & 53 & 54 & 1 & 716 & 71 & 70 & & & & & \\
\hline
\end{tabular}

